Research Article

\title{
Generation and Modified Projective Synchronization for a Class of New Hyperchaotic Systems
}

\author{
Nuo Jia and Tao Wang \\ School of Mathematical Sciences, Harbin Normal University, Harbin 150025, China \\ Correspondence should be addressed to Tao Wang; wangtaohrb@gmail.com
}

Received 24 November 2012; Revised 7 March 2013; Accepted 13 March 2013

Academic Editor: Tianshou Zhou

Copyright ( 2013 N. Jia and T. Wang. This is an open access article distributed under the Creative Commons Attribution License, which permits unrestricted use, distribution, and reproduction in any medium, provided the original work is properly cited.

\begin{abstract}
A class of new hyperchaotic systems with different nonlinear terms is proposed, and the existence of hyperchaos is exhibited by calculating their Lyapunov exponent spectrums. Then the universal theories on modified projective synchronization (MPS) of the systems with general form which linearly depends on unknown parameters or time-varying parameters, are investigated by presenting an adaptive control strategy together with parameter update laws and a nonlinear control scheme based on Lyapunov stability theory. Subsequently, the presented control methods are applied to achieve MPS of the new hyperchaotic systems, and their effectiveness is illustrated by numerical simulations.
\end{abstract}

\section{Introduction}

Since the pioneer work by Pecora and Corroll in 1990 [1], chaos synchronization, which refers to a process wherein two (or many) chaotic systems (either equivalent or nonequivalent) adjust a given property of their motion to a common behavior due to a coupling or to a forcing (periodical or noisy) [2], has become an active research subject for its extensive potential application in physics, secure communication, chemical reactor, biological networks, and so on. Up to now, many different types of synchronization have been presented such as complete synchronization [1], phase synchronization [3], lag synchronization [4], generalized synchronization [5], and projective synchronization [6]. Among them, projective synchronization is the most noticeable one with the essence that the drive and response systems could be synchronized up to a scaling factor $\alpha$ (a proportional relation), because it has some topological invariants, such as Lyapunov exponents (LEs) and fractional dimensions which is understood well, and it could be used to extend binary digital to variety Mary digital communications for getting more secure and faster communications. By the way, generalized projective synchronization is its extension in general classes of chaotic systems including nonpartially linear systems [7]. Recently, a new synchronization termed as modified projective synchronization (MPS) [8] was presented, of which the different scaling factors in a scaling matrix $H$ can be arbitrarily designed to different state variables. It can be seen that MPS encompasses the complete synchronization, antisynchronization, and projective synchronization when scaling matrix $H$ equals to $I$, $-I$, and $\alpha I$ ( $\alpha$ is a constant), respectively. Consequently, it has more broad prospect in practical applications.

A lot of work has been done around these different chaos synchronization phenomena, which can be summarized to two aspects as generation of chaotic systems and synchronization schemes to achieve chaos synchronization. On the one hand, since Rössler first introduced the hyperchaotic dynamical system in 1979 [9], some hyperchaotic systems are constructed by adding state feedback to 3D chaotic systems such as Lorenz system, Chen system, and Lü system, and have been investigated to some degree [10-13]. In comparison with low-dimensional chaotic system, hyperchaotic system with higher than or equal to four dimension has two or more positive Lyapunov exponents, richer and more complex dynamical behaviors which appears in more directional separation of phase orbits, and much wider application. So, from a practical point of view, some scholars devote to applying hyperchaotic system to generate more unpredictable and noise-like chaotic signals and considering synchronization between two hyperchaotic systems or between chaotic system and hyperchaotic system [14-20]. However, it is still an interesting task to derive $4 \mathrm{D}$ or higher-dimensional hyperchaotic systems. 
On the other hand, various synchronization schemes have been proposed such as linear and nonlinear feedback synchronization method [21-24], adaptive synchronization method [25-31], time-delay feedback method [32, 33], backstepping control method [34,35], sliding mode control method [36, 37], and impulsive synchronization method [38, 39]. Among them, adaptive control and nonlinear control methods are often used to solve the problems on synchronization of systems with unknown parameters or time-varying parameters, which are usually encountered in practical applications. However, most of them mentioned above have concentrated on achieving complete synchronization of lowdimensional and identical chaotic systems, while synchronization schemes for identical or nonidentical hyperchaotic systems have not been investigated extensively enough. As far as we know within our range, there is few literatures on MPS of nonidentical hyperchaotic systems. Therefore, designing effective control schemes to achieve MPS of two hyperchaotic systems with unknown parameters or time-varying parameters is an interesting and challenging job for both theory and practical applications.

Motivated by the aforementioned aspects, we first propose a class of new systems with different nonlinear terms and show the existence of hyperchaos in certain parameter ranges by calculating their Lyapunov exponent spectrums. After that, by presenting an adaptive control strategy and a nonlinear control scheme based on Lyapunov stability theory, the theories on MPS of the systems with general form which linearly depends on unknown parameters or time-varying parameters, respectively, are investigated. Finally, the presented control methods are applied to achieve MPS of the new hyperchaotic systems, and their effectiveness is illustrated by numerical simulations. The organization of this paper is as follows. In Section 2, a class of new hyperchaotic systems is constructed and the existence of hyperchaos is shown. In Section 3, theories on MPS of the systems with general form are given. At last, MPS of our presented hyperchaotic systems together with numerical simulations is shown in Section 4.

\section{The Description of a Class of New Hyperchaotic Systems}

There are two important requisites to obtain hyperchaos. One that is the minimal dimension of the phase space that embeds a hyperchaotic attractor should be at least four, and the other that is the number of terms in the coupled equations giving rise to instability should be at least two, of which at least one should have a nonlinear function [9]. According to the two points, a class of new 4D hyperchaotic systems is proposed by modifying the nonlinear terms of the Lorenz system and adding state feedback to it. They are described as

$$
\begin{gathered}
\dot{x}_{1}=a\left(x_{2}-x_{1}\right), \\
\dot{x}_{2}=b x_{1}-10 x_{1} x_{3}+x_{2}+x_{4}, \\
\dot{x}_{3}=-c x_{3}+10 T\left(x_{1}, x_{2}\right), \\
\dot{x}_{4}=-d Q\left(x_{1}, x_{2}, x_{3}, x_{4}\right)+R\left(x_{1}, x_{2}, x_{3}, x_{4}\right),
\end{gathered}
$$

TABLE 1: New hyperchaotic systems.

\begin{tabular}{lccc}
\hline System & $T$ & $Q$ & $R$ \\
\hline (a) & $x_{1}^{2}$ & $x_{2}$ & $x_{2} x_{4}$ \\
(b) & $x_{1} x_{2}$ & $x_{2}$ & $x_{1} x_{3}$ \\
(c) & $x_{1} x_{2}$ & $x_{2}$ & $x_{2} x_{4}$ \\
(d) & $x_{1}^{2}$ & $x_{2}$ & $x_{1} x_{3}$ \\
(e) & $x_{1}^{2}$ & $x_{1}$ & $x_{1} x_{3}$ \\
(f) & $x_{1} x_{2}$ & $x_{1}$ & $x_{1} x_{3}$ \\
\hline
\end{tabular}

where $a, b, c$, and $d$ are parameters to be tuned and $x_{1}$, $x_{2}, x_{3}$, and $x_{4}$ are state variables. $T\left(x_{1}, x_{2}\right), R\left(x_{1}, x_{2}, x_{3}, x_{4}\right)$ are nonlinear continuous functions, and $Q\left(x_{1}, x_{2}, x_{3}, x_{4}\right)$ is a linear continuous function, which can be set to obtain different hyperchaotic systems. We set $T, R$ as nonlinear quadratic functions and $Q$ as $x_{1}, x_{2}, x_{3}$, or $x_{4}$ to show systems with relatively simple forms. Subsequently, six different nonlinear systems are listed in Table 1.

In the following, the dynamics of the system (a) with $T=$ $x_{1}^{2}, Q=x_{2}$, and $R=x_{2} x_{4}$ is illustrated as an example, which is described as

$$
\begin{gathered}
\dot{x}_{1}=a\left(x_{2}-x_{1}\right), \\
\dot{x}_{2}=b x_{1}-10 x_{1} x_{3}+x_{2}+x_{4}, \\
\dot{x}_{3}=-c x_{3}+10 x_{1}^{2}, \\
\dot{x}_{4}=-d x_{2}+x_{2} x_{4} .
\end{gathered}
$$

In case of $a=20, b=35, c=3$, and $d=10$, it has four equilibrium points, and the types of which can be determined by calculating the eigenvalues of the Jacobian matrices, respectively. The detailed descriptions are shown in Table 2, where USNP and USFP mean unstable saddle-node point and unstable saddle-focus point, respectively.

Furthermore, the chaotic attractor is shown in Figure 1. Combined with calculated Lyapunov Exponents (LEs) $\lambda_{1}=$ 1.0677, $\lambda_{2}=0.0994, \lambda_{3}=0, \lambda_{4}=-23.1526$, and Lyapunov Dimension (LD) 3.0498, respectively, we can say that system (2) is hyperchaotic. These analyses suggest that system (2) has rich dynamics with fixed parameters. In order to give the existence of chaos and hyperchaos in different parameter ranges, the Lyapunov exponent spectrum versus parameters $a, b, c$, and $d$ for the first three LEs $\lambda_{1}, \lambda_{2}$, and $\lambda_{3}$ is shown, respectively, in Figure 2, where it can be clearly seen how it evolves from negative to positive values. It is noted that the system (2) is hyperchaotic when $b \in(12,75)$ and chaotic when $b \in(75,2000)$. The Largest Lyapunov exponent is $\lambda=$ 10.105 when $b=2000$, which suggests it has a big chaotic range and complex dynamics.

The similar analyses for the other five systems can also be gotten naturally. To highlight the existence of hyperchaos, we only exhibit the Lyapunov exponent spectrums of the systems (b)-(f) versus $b$ for $\lambda_{1}, \lambda_{2}$, and $\lambda_{3}$ when $a=20, c=3$, and $d=10$ in Figure 3. It can be concluded from Figures 2 and 3 that the six new different systems are all hyperchaotic when $b \in(20,60)$. 

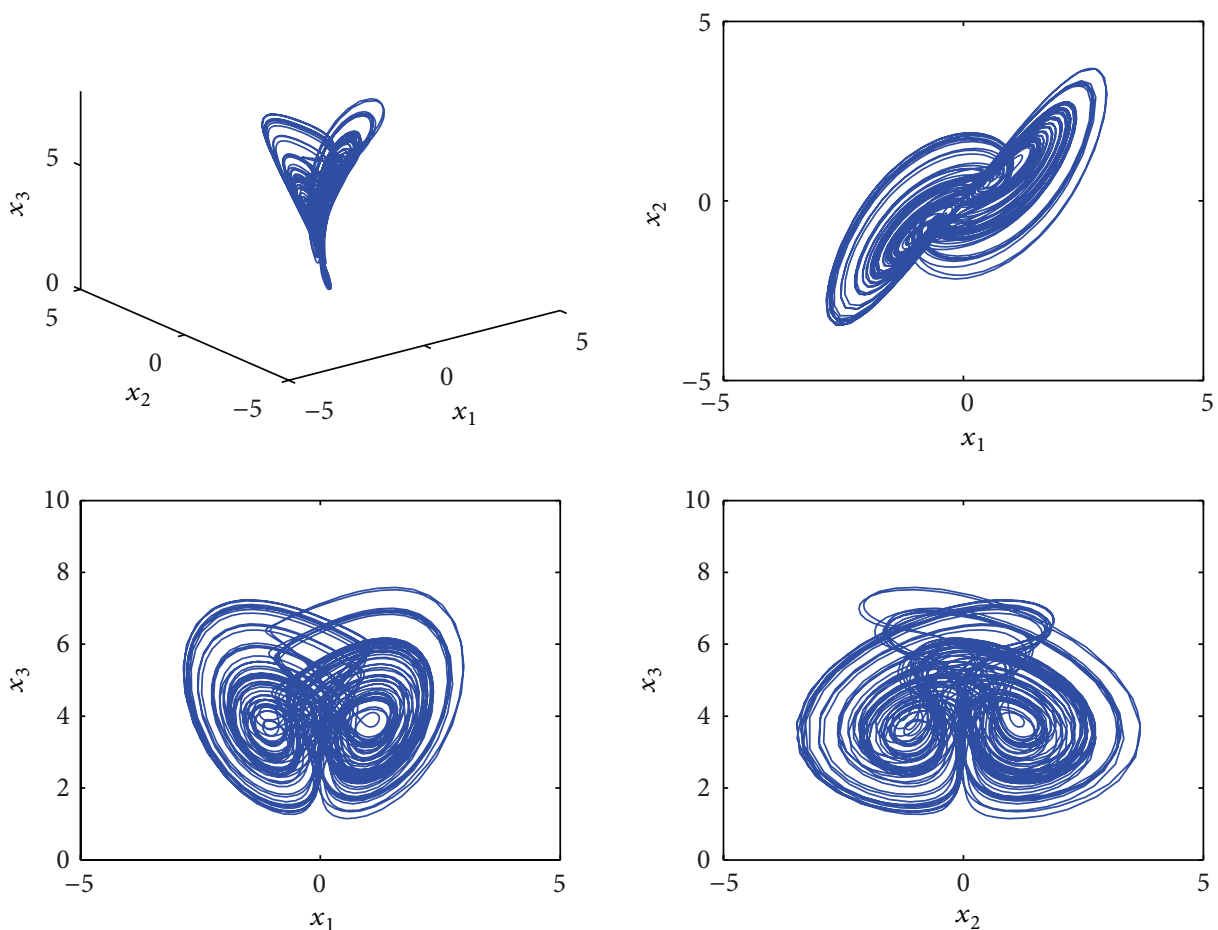

FIGURE 1: The chaotic attractor of system (2) with $a=20, b=35, c=3$, and $d=10$.
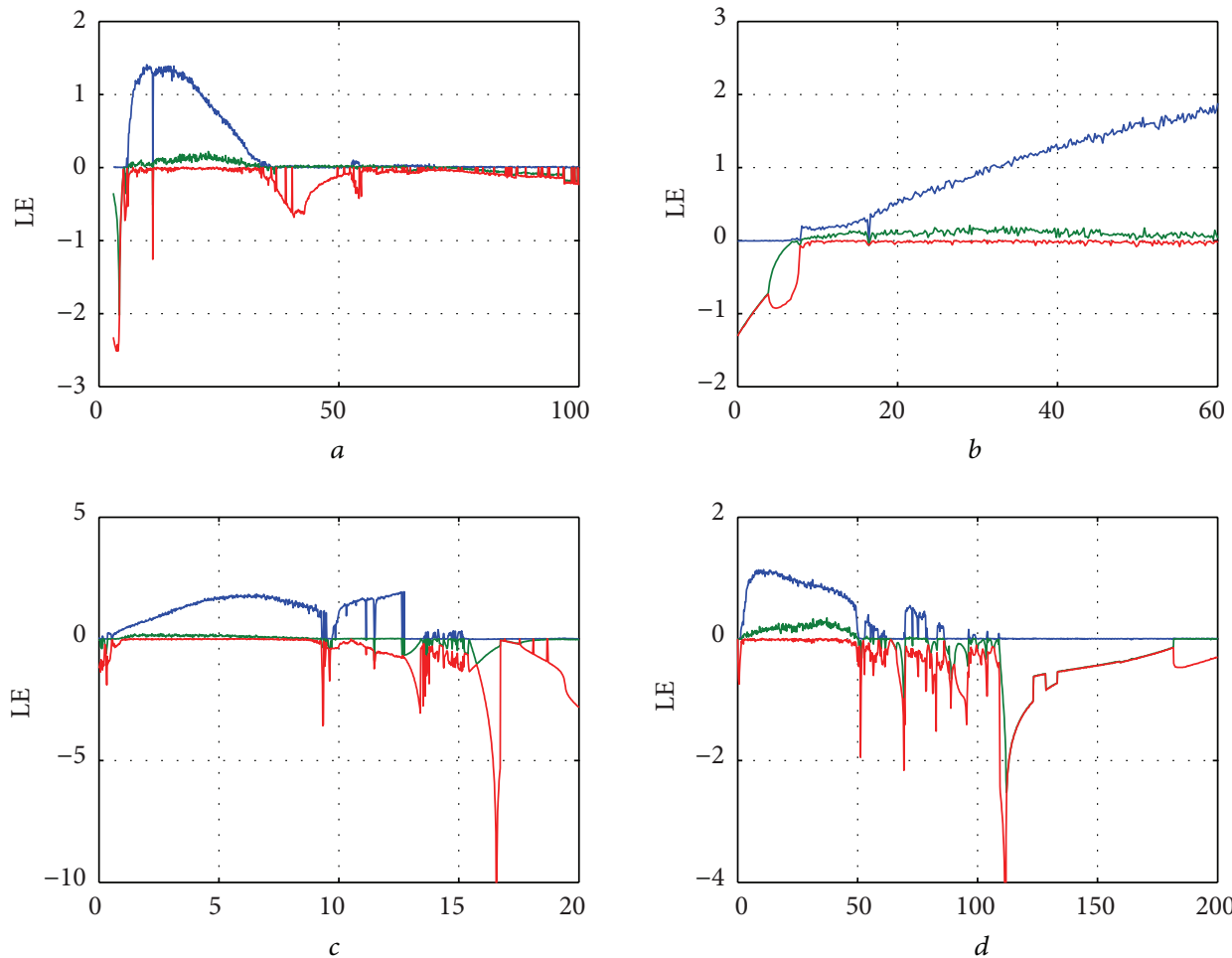

FIGURE 2: The Lyapunov exponent spectrum of system (2) versus parameters $a, b, c$, and $d$ for the first three LEs. 
TABLE 2: The related descriptions on the equilibrium points of system (2) with $a=20, b=35, c=3$, and $d=10$.

\begin{tabular}{lcc}
\hline Equilibrium points & Eigenvalues of Jacobian matrices & Equilibrium point types \\
\hline$P_{0}(0,0,0,0)$ & $-37.8819,18.5980,0.2839,-3$ & USNP \\
$P_{1}(1.1573,1.1573,4.4641,10)$ & $-23.0754,0.5377 \pm 15.9483 i, 1.1573$ & USFP \\
$P_{2}(-0.3037,-0.3037,0.3075,10)$ & $-37.0605,17.5330,-2.4725,-0.3037$ & USNP \\
$P_{3}(-0.8535,-0.8535,2.4284,10)$ & $-30.2706,4.1353 \pm 7.4791 i,-0.8535$ & USFP \\
\hline
\end{tabular}
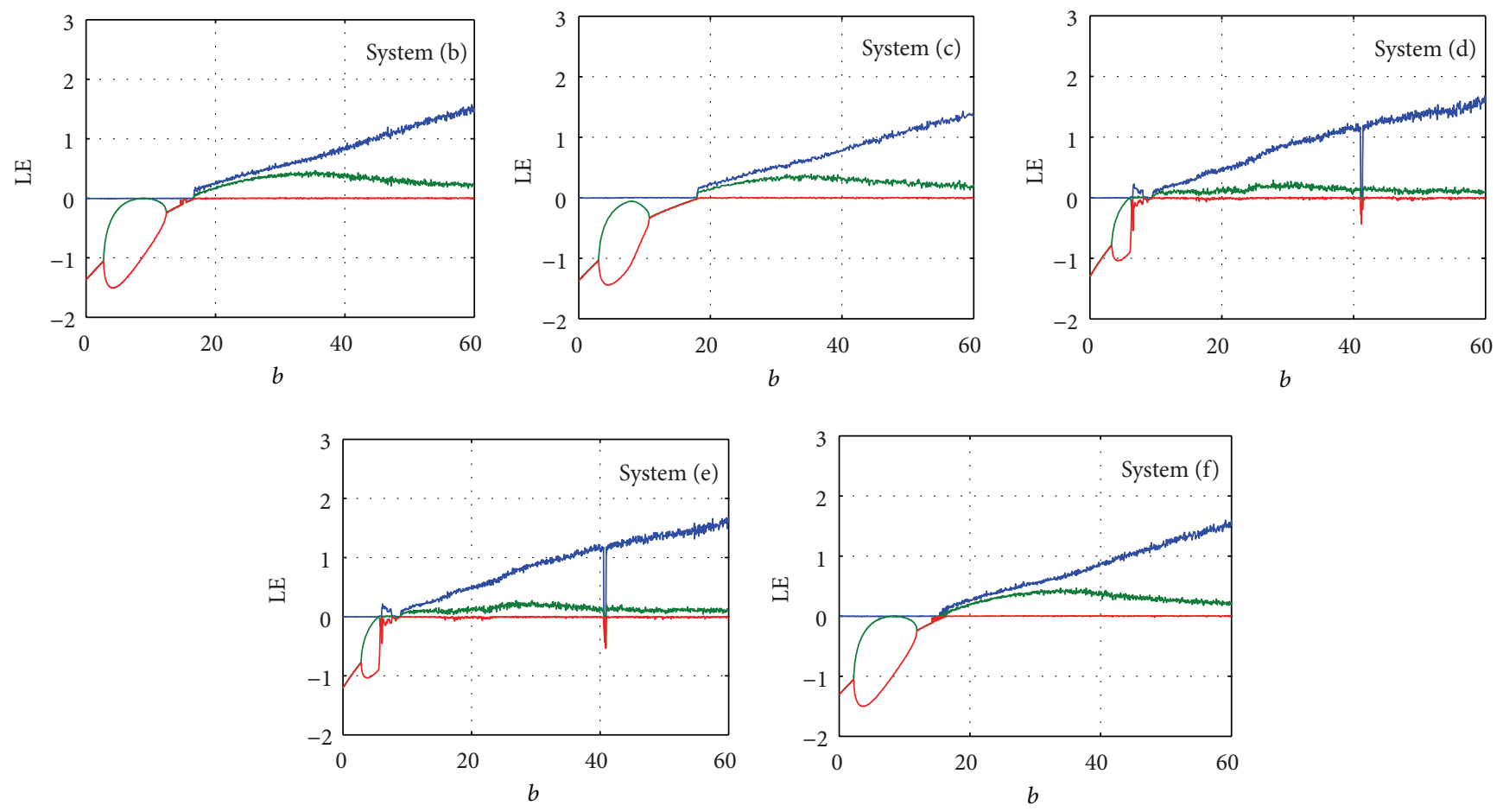

FIGURE 3: The Lyapunov exponent spectrum of system (b)-(f) versus $b$ for $\lambda_{1}, \lambda_{2}$, and $\lambda_{3}$ when $a=20, c=3$, and $d=10$.

\section{Modified Projective Synchronization of General Chaotic Systems with the Same Structure to the New Hyperchaotic Systems}

3.1. The Preliminaries. Consider the following drive-response systems

$$
\begin{gathered}
\dot{x}=f(x), \\
\dot{y}=g(y)+u(t, x, y),
\end{gathered}
$$

where $x=\left(x_{1}, x_{2}, \ldots, x_{n}\right)^{T} \in R^{n}, y=\left(y_{1}, y_{2}, \ldots, y_{n}\right)^{T} \in R^{n}$ are state variables of the drive system (3) and the response system (4), respectively, $f: R^{n} \rightarrow R^{n}$ and $g: R^{n} \rightarrow R^{n}$ are continuous nonlinear vector functions, and $u(t, x, y)=$ $\left(u_{1}, u_{2}, \ldots, u_{n}\right)^{T} \in R^{n}$ is the control vector for synchronization.

Definition 1. For the drive system (3) and the response system (4), they are said to be modified projective synchronization (MPS) if there exists a nonzero constant matrix $H=$ $\operatorname{diag}\left(h_{1}, h_{2}, \ldots, h_{n}\right) \in R^{n \times n}$, such that $\lim _{t \rightarrow \infty}\|y-H x\|=0$, namely, $\lim _{t \rightarrow \infty}\left|y_{i}-h_{i} x_{i}\right|=0(i=1,2, \ldots, n)$, where $H$ is scaling matrixand $h_{1}, h_{2}, \ldots, h_{n}$ are nonzero scaling factors which could be a predefined value or any desired value to be directed by a feedback control.

Remark 2. When the scaling matrix $H$ equals to $I,-I$, and $\alpha I$ ( $\alpha$ is a constant), respectively, it means complete synchronization, antisynchronization, and projective synchronization, respectively.

Aiming at considering MPS between two of the systems (a)-(f), we first investigate the theories on MPS of general chaotic systems with the same structure to them in this part. Consider an $n$-dimensional continuous chaotic (hyperchaotic) system as drive system in the form of

$$
\dot{x}=f(x)+F(x) \theta_{1},
$$

where $x=\left(x_{1}, x_{2}, \ldots, x_{n}\right)^{T} \in R^{n}$ is the state vector, $f: R^{n} \rightarrow$ $R^{n}$ is a continuous nonlinear vector function, $F: R^{n} \rightarrow R^{n \times n}$ is a continuous function matrix, and $\theta_{1} \in R^{n}$ is a parameter vector. It can be seen that the nonlinear dynamical system (5) 
linearly depends on the parameter vector, and systems (a)(f) all have the same system structure, so do many wellknown hyperchaotic systems, such as hyperchaotic Lorenz, Lü systems, and Rössler system. Accompanied with the drive system (5), a controlled response system is given by

$$
\dot{y}=g(y)+G(y) \theta_{2}+u,
$$

where $y=\left(y_{1}, y_{2}, \ldots, y_{n}\right)^{T} \in R^{n}$ is the state vector, $g$ : $R^{n} \rightarrow R^{n}$ is a continuous vector function, $G: R^{n} \rightarrow R^{n \times n}$ is a continuous function matrix, $\theta_{2} \in R^{n}$ is a parameter vector, and $u \in R^{n}$ is the control vector to be determined. Let $e=y-H x$ denote the error state vector, thus the error dynamical system has the form

$$
\begin{aligned}
\dot{e} & =\dot{y}-H \dot{x}=g(y)+G(y) \theta_{2}-H f(x)-H F(x) \theta_{1}+u \\
& =Q(e, x)+G(e, x) \theta_{2}-H F(x) \theta_{1}+u,
\end{aligned}
$$

where $Q(e, x)=g(e+H x)-H f(x)$ and $H=\operatorname{diag}\left(h_{1}, h_{2}\right.$, $\left.\ldots, h_{n}\right)$. So the global and asymptotical stability of system (7) means that systems (5) and (6) achieve MPS.

3.2. MPS between Systems (5) and (6) with Unknown or Time-Varying Parameters. Usually, the system parameters are partially or entirely unknown in advance in practical applications, and adaptive controller is often used to solve the problem for its adaptive ability. So one of our objects is to design an adaptive synchronization scheme with parameter update laws

$$
\begin{gathered}
u=u\left(x, y, \widehat{\theta}_{1}, \widehat{\theta}_{2}\right), \quad \dot{\hat{\theta}}_{1}=\theta_{1}\left(x, y, \widehat{\theta}_{1}, \widehat{\theta}_{2}\right), \\
\dot{\hat{\theta}}_{2}=\theta_{2}\left(x, y, \widehat{\theta}_{1}, \widehat{\theta}_{2}\right),
\end{gathered}
$$

where $\hat{\theta}_{1}$ and $\hat{\theta}_{2}$ are parameter estimate vectors of the unknown parameter vectors $\theta_{1}, \theta_{2}$, to get the drive-response systems to be in MPS with any arbitrarily given scaling factors. Namely, $\|y-H x\| \rightarrow 0$, together with $\widehat{\theta}_{1} \rightarrow \theta_{1}, \widehat{\theta}_{2} \rightarrow$ $\theta_{2}$ for $t \rightarrow \infty$.

Theorem 3. For given nonzero scaling factors $h_{i} \neq 0 \quad(i=$ $1,2, \ldots, n)$, the drive-response systems (5) and (6) achieve MPS if the control vector and the parameter update laws are given as

$$
\begin{gathered}
u=-Q(e, x)-G(e, x) \hat{\theta}_{2}+H F(x) \hat{\theta}_{1}-M e, \\
\dot{\bar{\theta}}_{1}=-\bar{\theta}_{1}+F^{T}(x) H e \\
\dot{\bar{\theta}}_{2}=-\bar{\theta}_{2}-G^{T}(e, x) e
\end{gathered}
$$

where $\bar{\theta}_{i}=\theta_{i}-\widehat{\theta}_{i}, i=1,2$, and $M$ is a known positive definite matrix.

Proof. Substitute (9) into the error system (7), we get

$$
\dot{e}=-M e-H F(x) \bar{\theta}_{1}+G(e, x) \bar{\theta}_{2} .
$$

Construct a Lyapunov function

$$
V=\frac{1}{2} e^{T} e+\frac{1}{2} \bar{\theta}_{1}^{T} \bar{\theta}_{1}+\frac{1}{2} \bar{\theta}_{2}^{T} \bar{\theta}_{2}
$$

and differentiate $V$ with respect to time along the solution of (12). It yields

$$
\begin{aligned}
\dot{V}(t)= & e^{T} \dot{e}+\dot{\bar{\theta}}_{1}^{T} \bar{\theta}_{1}+\dot{\bar{\theta}}_{2}^{T} \bar{\theta}_{2} \\
= & e^{T}\left(-M e-H F(x) \bar{\theta}_{1}+G(e, x) \bar{\theta}_{2}\right) \\
& +\left(-\bar{\theta}_{1}+F^{T}(x) H e\right)^{T} \bar{\theta}_{1} \\
& +\left(-\bar{\theta}_{2}-G^{T}(e, x) e\right)^{T} \bar{\theta}_{2} \\
= & -M e^{T} e-\bar{\theta}_{1}^{T} \bar{\theta}_{1}-\bar{\theta}_{2}^{T} \bar{\theta}_{2} \\
\leq & 0,
\end{aligned}
$$

namely, $\dot{V}$ is negative definite. It results in that the driveresponse systems (5) and (6) achieve MPS according to Lyapunov stability theorem.

The other object is to achieve MPS between chaotic systems with time-varying parameters which are also frequently encountered in practical applications. Suppose parameter vectors $\theta_{1}=\theta_{1}(t), \theta_{2}=\theta_{2}(t)$ of drive-response systems are both time varying and bounded, which means if one denote $\widetilde{\theta}_{1}, \widetilde{\theta}_{2}$ the nominal constant vectors of $\theta_{1}, \theta_{2}$, respectively, and $\Theta_{1}, \Theta_{2}$ known upper bounds, then

$$
\left\|\theta_{1}-\widetilde{\theta}_{1}\right\| \leq \Theta_{1}, \quad\left\|\theta_{2}-\widetilde{\theta}_{2}\right\| \leq \Theta_{2} .
$$

In addition, since as far as we know, most hyperchaotic systems in the existing literatures such as hyperchaotic Lorenz system, hyperchaotic Lü system, and Rössler system, as well as the class of new systems (1) proposed here have the vectorial form (5) with diagonal $F(x)$, MPS of this kind of general hyperchaotic systems is discussed in the following theorem. It is noted that $\bar{A}=\left(\left|a_{i j}\right|\right)_{n \times n}$ denotes a matrix, each element of which is the absolute value of corresponding element of matrix $A=\left(a_{i j}\right)_{n \times n}$, and $A_{i i}$ denotes its element at the cross of the $i$ th row and the $i$ th column.

Theorem 4. For given nonzero scaling factors $h_{i} \neq 0 \quad(i=$ $1,2, \ldots, n)$, the drive-response systems (5) and (6) with timevarying parameters and diagonal $F(x)$ and $G(e, x)$ can achieve $M P S$ if the nonlinear control strategy is designed as

$$
\begin{aligned}
u= & -P e-Q(e, x)-G(e, x) \widetilde{\theta}_{2}+H F(x) \widetilde{\theta}_{1} \\
& -\bar{G}(e, x) \Theta_{2} \operatorname{sgn}(e)-\overline{H F}(x) \Theta_{1} \operatorname{sgn}(e),
\end{aligned}
$$

where $P$ is a known positive definite matrix and $\operatorname{sgn}(e)$ denote a vector with elements $\operatorname{sgn}\left(e_{i}\right), i=1,2, \ldots, n$.

Proof. According to (16), we rewrite the error system (7) as

$$
\begin{aligned}
\dot{e}= & -P e-H F(x)\left(\theta_{1}-\widetilde{\theta}_{1}\right)+G(e, x)\left(\theta_{2}-\widetilde{\theta}_{2}\right) \\
& -\bar{G}(e, x) \Theta_{2} \operatorname{sgn}(e)-\overline{H F}(x) \Theta_{1} \operatorname{sgn}(e) .
\end{aligned}
$$


Constructing a Lyapunov function $V=e^{T} e / 2$ and differentiate $V$ with respect to time along the solution of (17), we have

$$
\begin{aligned}
\dot{V}(t)= & e^{T} \dot{e} \\
= & e^{T}\left(-P e-H F(x)\left(\theta_{1}-\widetilde{\theta}_{1}\right)+G(e, x)\left(\theta_{2}-\widetilde{\theta}_{2}\right)\right. \\
& \left.\quad-\bar{G}(e, x) \Theta_{2} \operatorname{sgn}(e)-\overline{H F}(x) \Theta_{1} \operatorname{sgn}(e)\right) \\
= & -e^{T} P e-e^{T} H F(x)\left(\theta_{1}-\widetilde{\theta}_{1}\right)+e^{T} G(e, x)\left(\theta_{2}-\widetilde{\theta}_{2}\right) \\
& -e^{T} \bar{G}(e, x) \Theta_{2} \operatorname{sgn}(e)-e^{T} \overline{H F}(x) \Theta_{1} \operatorname{sgn}(e) \\
\leq & -e^{T} P e+\left|e^{T} H F(x)\left(\theta_{1}-\widetilde{\theta}_{1}\right)\right|-e^{T} \overline{H F}(x) \Theta_{1} \operatorname{sgn}(e) \\
& +\left|e^{T} G(e, x)\left(\theta_{2}-\widetilde{\theta}_{2}\right)\right|-e^{T} \bar{G}(e, x) \Theta_{2} \operatorname{sgn}(e) \\
\leq & -e^{T} P e+\bar{e}^{T} \overline{H F}(x) \Theta_{1} \\
& -e^{T} \overline{H F}(x) \Theta_{1} \operatorname{sgn}(e)+\bar{e}^{T} \bar{G}(e, x) \Theta_{2} \\
& -e^{T} \bar{G}(e, x) \Theta_{2} \operatorname{sgn}(e) .
\end{aligned}
$$

It results in $\dot{V}=-e^{T} P e \leq 0$ because

$$
\begin{aligned}
e^{T} \overline{H F}(x) \Theta_{1} \operatorname{sgn}(e) & =\Theta_{1} \sum_{i=1}^{n} \bar{H}_{i i} \bar{F}(x)_{i i} e_{i} \operatorname{sgn}(e) \\
& =\Theta_{1} \sum_{i=1}^{n} \bar{H}_{i i} \bar{F}(x)_{i i}\left|e_{i}\right|=\bar{e}^{T} \overline{H F}(x) \Theta_{1}, \\
e^{T} \bar{G}(e, x) \Theta_{2} \operatorname{sgn}(e) & =\Theta_{2} \sum_{i=1}^{n} \bar{G}(e, x)_{i i} e_{i} \operatorname{sgn}\left(e_{i}\right) \\
& =\Theta_{2} \sum_{i=1}^{n} \bar{G}(e, x)_{i i}\left|e_{i}\right|=\bar{e}^{T} \bar{G}(e, x) \Theta_{2} .
\end{aligned}
$$

Based on Lyapunov stability theory, the system (17) converges to $O(0,0,0,0)$ as $t \rightarrow \infty$, which means that the two hyperchaotic systems achieve MPS asymptotically. This completes the proof.

\section{MPS of the New Hyperchaotic Systems and Numerical Simulations}

The presented theories are applied to MPS between two of the new hyperchaotic systems in this part. Set system (c) and system (f) as drive-response systems, which have the forms

$$
\begin{gathered}
\dot{x}_{1}=a_{1}\left(x_{2}-x_{1}\right), \\
\dot{x}_{2}=b_{1} x_{1}-10 x_{1} x_{3}+x_{4}+x_{2}, \\
\dot{x}_{3}=-c_{1} x_{3}+10 x_{1} x_{2}, \\
\dot{x}_{4}=-d_{1} x_{2}+x_{2} x_{4},
\end{gathered}
$$

$$
\begin{gathered}
\dot{y}_{1}=a_{2}\left(y_{2}-y_{1}\right)+u_{1}, \\
\dot{y}_{2}=b_{2} y_{1}-10 y_{1} y_{3}+y_{4}+y_{2}+u_{2}, \\
\dot{y}_{3}=-c_{2} y_{3}+10 y_{1} y_{2}+u_{3}, \\
\dot{y}_{4}=-d_{2} y_{1}+y_{1} y_{3}+u_{4},
\end{gathered}
$$

where $a_{1}, b_{1}, c_{1}$, and $d_{1}$ and $a_{2}, b_{2}, c_{2}$, and $d_{2}$ are unknown system parameters which need to be estimated. Their vector forms can be, respectively, described as

$$
\begin{aligned}
\left(\begin{array}{c}
\dot{x}_{1} \\
\dot{x}_{2} \\
\dot{x}_{3} \\
\dot{x}_{4}
\end{array}\right)= & \left(\begin{array}{c}
0 \\
-10 x_{1} x_{3}+x_{4}+x_{2} \\
10 x_{1} x_{2} \\
x_{2} x_{4}
\end{array}\right) \\
& +\left(\begin{array}{cccc}
x_{2}-x_{1} & 0 & 0 & 0 \\
0 & x_{1} & 0 & 0 \\
0 & 0 & -x_{3} & 0 \\
0 & 0 & 0 & -x_{2}
\end{array}\right)\left(\begin{array}{l}
a_{1} \\
b_{1} \\
c_{1} \\
d_{1}
\end{array}\right),
\end{aligned}
$$

$$
\begin{aligned}
\left(\begin{array}{c}
\dot{y}_{1} \\
\dot{y}_{2} \\
\dot{y}_{3} \\
\dot{y}_{4}
\end{array}\right)= & \left(\begin{array}{c}
0 \\
-10 y_{1} y_{3}+y_{4}+y_{2} \\
10 y_{1} y_{2} \\
y_{1} y_{3}
\end{array}\right) \\
& +\left(\begin{array}{cccc}
y_{2}-y_{1} & 0 & 0 & 0 \\
0 & y_{1} & 0 & 0 \\
0 & 0 & -y_{3} & 0 \\
0 & 0 & 0 & -y_{1}
\end{array}\right)\left(\begin{array}{l}
a_{2} \\
b_{2} \\
c_{2} \\
d_{2}
\end{array}\right) \\
& +\left(\begin{array}{l}
u_{1} \\
u_{2} \\
u_{3} \\
u_{4}
\end{array}\right),
\end{aligned}
$$

where $\left(u_{1}, u_{2}, u_{3}, u_{4}\right)^{T}$ is the controller to be determined. Let positive definite matrix $M$ be

$$
M=\left(\begin{array}{llll}
1 & 0 & 0 & 0 \\
0 & 1 & 0 & 1 \\
0 & 0 & 1 & 0 \\
0 & 0 & 0 & 1
\end{array}\right)
$$

then according to (9), (10), and (11), we get the controller

$$
\begin{aligned}
u_{1}=-e_{1}- & \dot{\hat{a}}_{2}\left(e_{2}-e_{1}+h_{2} x_{2}-h_{1} x_{1}\right)+\dot{\hat{a}}_{1} h_{1}\left(x_{2}-x_{1}\right), \\
u_{2}= & -2 e_{2}-e_{4}+10\left(e_{1}+h_{1} x_{1}\right)\left(e_{3}+h_{3} x_{3}\right) \\
& -\left(e_{4}+h_{4} x_{4}\right)-10 h_{2} x_{1} x_{3} \\
& +h_{2} x_{4}-\dot{\hat{b}}_{2}\left(e_{1}+h_{1} x_{1}\right)+\dot{\hat{b}}_{1} h_{2} x_{1},
\end{aligned}
$$




$$
\begin{aligned}
u_{3}= & -e_{3}-10\left(e_{1}+h_{1} x_{1}\right)\left(e_{2}+h_{2} x_{2}\right) \\
& +10 h_{3} x_{1} x_{2}+\dot{\hat{c}}_{2}\left(e_{3}+h_{3} x_{3}\right)-\dot{\hat{c}}_{1} h_{3} x_{3}, \\
u_{4}= & -e_{4}-\left(e_{1}+h_{1} x_{1}\right)\left(e_{3}+h_{3} x_{3}\right) \\
& +h_{4} x_{2} x_{4}+\dot{\hat{d}}_{2}\left(e_{1}+h_{1} x_{1}\right)-\dot{\hat{d}}_{1} h_{4} x_{2},
\end{aligned}
$$

with the parameter update laws

$$
\begin{gathered}
\dot{\bar{a}}_{1}=-\bar{a}_{1}+h_{1}\left(x_{2}-x_{1}\right) e_{1}, \\
\dot{\bar{b}}_{1}=-\bar{b}_{1}+h_{2} x_{1} e_{2}, \\
\dot{\bar{c}}_{1}=-\bar{c}_{1}-h_{3} x_{3} e_{3}, \\
\dot{\bar{d}}_{1}=-\bar{d}_{1}-h_{4} x_{2} e_{4}, \\
\dot{\bar{a}}_{2}=-\bar{a}_{2}-\left(e_{2}-e_{1}+h_{2} x_{2}-h_{1} x_{1}\right) e_{1}, \\
\dot{\bar{b}}_{2}=-\bar{b}_{2}-\left(e_{1}+h_{1} x_{1}\right) e_{2}, \\
\dot{\bar{c}}_{2}=-\bar{c}_{2}+\left(e_{3}+h_{3} x_{3}\right) e_{3}, \\
\dot{\bar{d}}_{2}=-d_{2}+\left(e_{1}+h_{1} x_{1}\right) e_{4},
\end{gathered}
$$

where $\bar{a}_{i}=a_{i}-\widehat{a}_{i}, \bar{b}_{i}=b_{i}-\widehat{b}_{i}, \bar{c}_{i}=c_{i}-\widehat{c}_{i}, \bar{d}_{i}=d_{i}-\widehat{d}_{i}$, and $i=1,2$. Let $a_{1}=a_{2}=20, b_{1}=b_{2}=35, c_{1}=c_{2}=3$, and $d_{1}=d_{2}=10$, then the drive-response systems are hyperchaotic. In addition, set the initial states of the drive-response systems to be $x_{1}(0)=1, x_{2}(0)=1, x_{3}(0)=1$, and $x_{4}(0)=1$ and $y_{1}(0)=4$, $y_{2}(0)=5, y_{3}(0)=6$, and $y_{4}(0)=7$, respectively, set the initial states of the estimated parameter errors to be $\bar{a}_{1}(0)=\bar{a}_{2}(0)=$ $1, \bar{b}_{1}(0)=\bar{b}_{2}(0)=1, \bar{c}_{1}(0)=\bar{c}_{2}(0)=1$, and $\bar{d}_{1}(0)=\bar{d}_{2}(0)=1$, and set the scaling matrix to be $H=\operatorname{diag}(-1,0.5,1,4)$. Then the time response of the errors is shown in Figure 4. For further observations, the state trajectories of the two systems are depicted in Figure 5. It is exhibited that $x_{1}$ and $y_{1}$ display an antisynchronization phenomenon, $y_{2}$ finally converges to half the value of $x_{2}, x_{3}$ and $y_{3}$ show synchronization behavior, and $y_{4}$ converges four times the value of $x_{4}$, just as we intended. Moreover, the curves of estimated parameters are also shown in Figure 6. It can be concluded that the two systems achieve MPS successfully.

Furthermore, suppose that the parameters are time varying, remain the drive system (20) and set system (e) to be response system which is expressed as

$$
\begin{gathered}
\dot{y}_{1}=a_{2}\left(y_{2}-y_{1}\right)+u_{1}, \\
\dot{y}_{2}=b_{2} y_{1}-10 y_{1} y_{3}+y_{4}+y_{2}+u_{2}, \\
\dot{y}_{3}=-c_{2} y_{3}+10 y_{1}^{2}+u_{3}, \\
\dot{y}_{4}=-d_{2} y_{1}+y_{1} y_{3}+u_{4},
\end{gathered}
$$
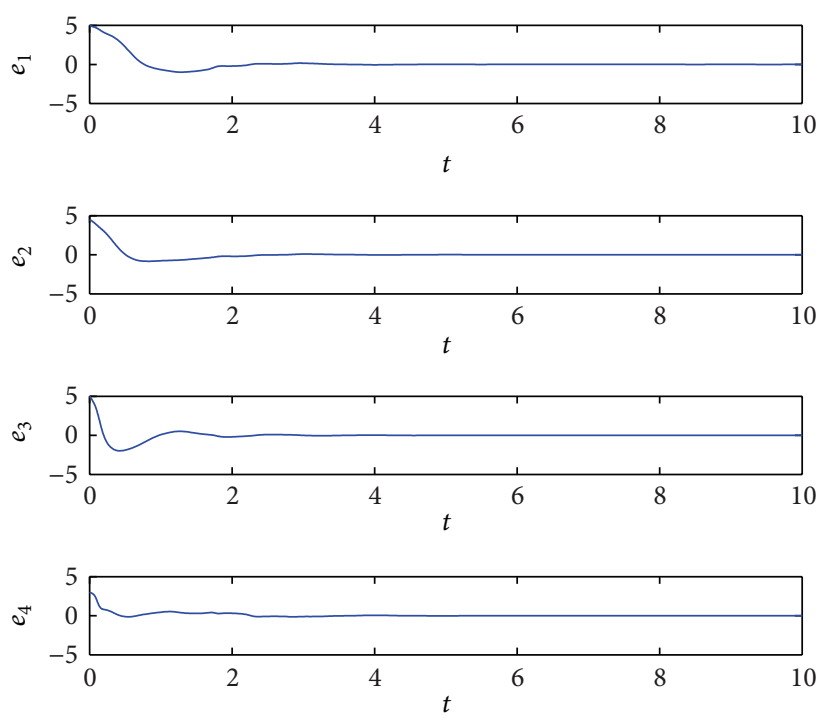

FIGURE 4: The MPS errors $e_{1}, e_{2}, e_{3}$, and $e_{4}$ between the driveresponse systems (20) and (21).

with the vector form

$$
\begin{aligned}
\left(\begin{array}{l}
\dot{y}_{1} \\
\dot{y}_{2} \\
\dot{y}_{3} \\
\dot{y}_{4}
\end{array}\right)= & \left(\begin{array}{c}
0 \\
-10 y_{1} y_{3}+y_{4}+y_{2} \\
10 y_{1}^{2} \\
y_{1} y_{3}
\end{array}\right) \\
& +\left(\begin{array}{cccc}
y_{2}-y_{1} & 0 & 0 & 0 \\
0 & y_{1} & 0 & 0 \\
0 & 0 & -y_{3} & 0 \\
0 & 0 & 0 & -y_{1}
\end{array}\right)\left(\begin{array}{c}
a_{2} \\
b_{2} \\
c_{2} \\
d_{2}
\end{array}\right) \\
& +\left(\begin{array}{c}
u_{1} \\
u_{2} \\
u_{3} \\
u_{4}
\end{array}\right),
\end{aligned}
$$

where $\left(u_{1}, u_{2}, u_{3}, u_{4}\right)^{T}$ is the controller to be determined. Set nominal values of $a, b, c$, and $d$ to be $a=20, b=35, c=3$, and $d=10$, set time-varying parameters of the drive-response systems to be $a_{1}=20+\sin (t), b_{1}=35+\sin (t), c_{1}=3+\sin (t)$, and $d_{1}=10+\sin (t)$ and $a_{2}=20+\cos (t), b_{2}=35+\cos (t)$, $c_{2}=3+\cos (t)$, and $d_{2}=10+\cos (t)$, respectively, set the upper bound to be $\Theta_{1}=\Theta_{2}=2$, set positive definite matrix to be $P=M$, set the initial states of the drive-response systems to be $x_{1}(0)=1, x_{2}(0)=1, x_{3}(0)=1$, and $x_{4}(0)=1$ and $y_{1}(0)=4, y_{2}(0)=5, y_{3}(0)=6, y_{4}(0)=7$, respectively, 

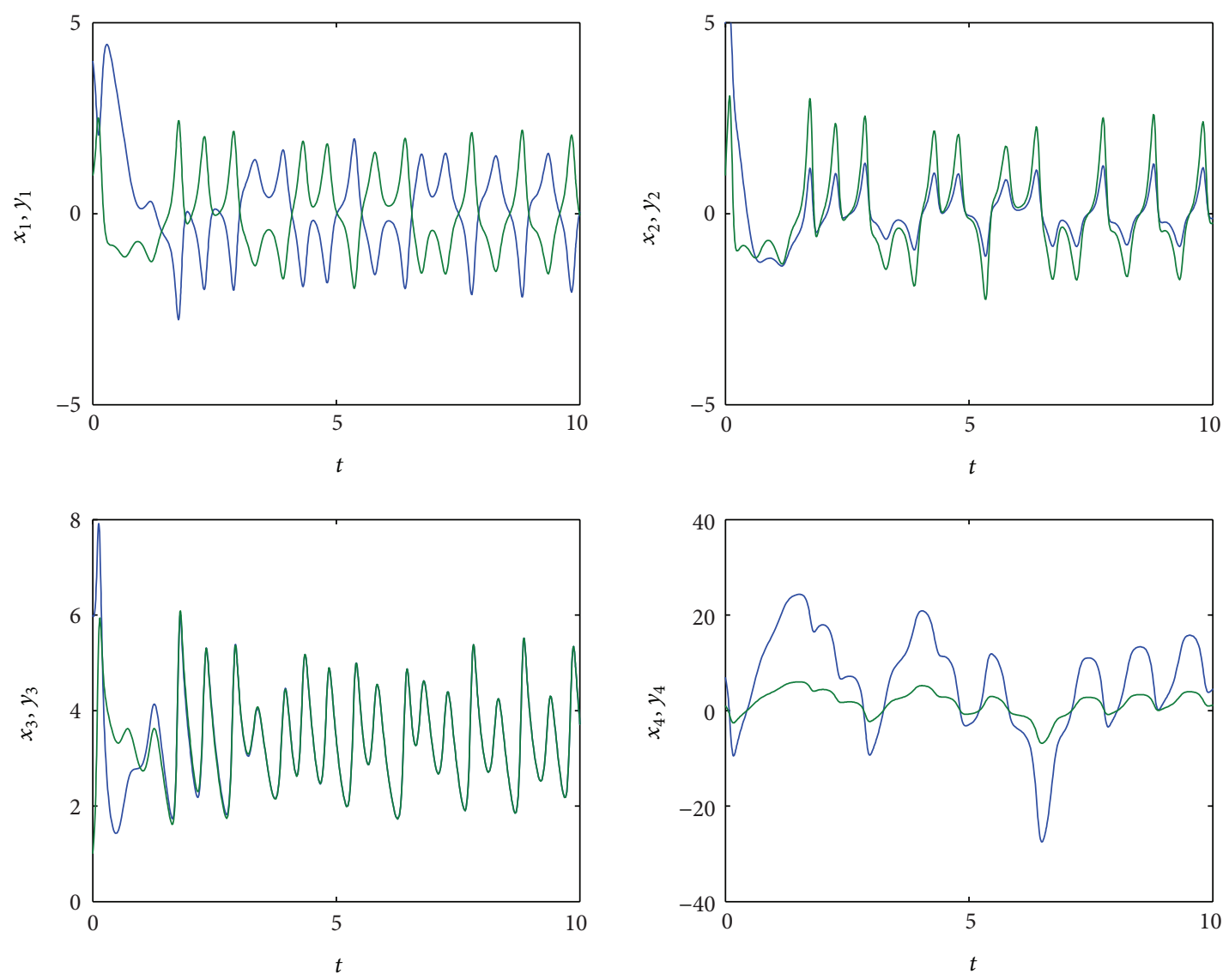

Figure 5: State trajectories of the drive-response systems (20) and (21), $x_{1}$ and $y_{1}$ with $h_{1}=-1, x_{2}$ and $y_{2}$ with $h_{2}=0.5, x_{3}$ and $y_{3}$ with $h_{3}=1$, and $x_{4}$ and $y_{4}$ with $h_{4}=4$.

and set the scaling matrix to be $H=\operatorname{diag}(-1,0.5,1,4)$, then according to (16), the controller can be described as

$$
\begin{aligned}
u_{1}= & -e_{1}-20\left(y_{2}-y_{1}\right)+20 h_{1}\left(x_{2}-x_{1}\right) \\
& -\left|y_{2}-y_{1}\right| \operatorname{sgn}\left(e_{1}\right)-\left|h_{1}\right|\left|x_{2}-x_{1}\right| \operatorname{sgn}\left(e_{1}\right), \\
u_{2}= & -2 e_{2}-e_{4}-10 y_{1} y_{3}+10 h_{2} x_{1} x_{3}-h_{2} x_{4}-h_{2} x_{2}-35 y_{1} \\
& +35 h_{2} x_{1}-\left|y_{1}\right| \operatorname{sgn}\left(e_{2}\right)-\left|h_{2}\right|\left|x_{1}\right| \operatorname{sgn}\left(e_{2}\right), \\
u_{3}= & -e_{3}-10 y_{1} y_{2}+10 h_{3} x_{1} x_{2}+3 y_{3}-3 h_{3} x_{3} \\
& +\left|y_{3}\right| \operatorname{sgn}\left(e_{3}\right)-\left|h_{3}\right|\left|x_{3}\right| \operatorname{sgn}\left(e_{3}\right), \\
u_{4}= & -e_{4}-y_{1} e_{3}+h_{4} x_{2} x_{4}+10 y_{1}-10 h_{4} x_{1} \\
& +\left|y_{1}\right| \operatorname{sgn}\left(e_{4}\right)-\left|h_{4}\right|\left|x_{2}\right| \operatorname{sgn}\left(e_{4}\right) .
\end{aligned}
$$

Subsequently, the time response of the error systems is given in Figure 7, which suggests, the error vector converges to zero asymptotically and the control strategy for MPS is successful.

\section{Conclusions}

Generation and MPS for a class of new hyperchaotic systems are both considered in this paper. First, six new hyperchaotic systems with different nonlinear terms are derived and the existence of hyperchaos is exhibited by calculating their Lyapunov exponent spectrums. Second, the universal theories on MPS of general chaotic systems with the structure like that are investigated by presenting an adaptive control strategy together with parameter update laws and a nonlinear control scheme based on Lyapunov stability theory. Finally, the methods are applied to our proposed hyperchaotic systems, and numerical simulations demonstrate the effectiveness of the proposed synchronization schemes.

\section{Acknowledgments}

The authors would like to thank the reviewer for his helpful suggestions on the paper. This research is supported by Natural Science Foundation of Heilongjiang Province, China (Grant no. A201101), the Science and Technology PreResearch Foundations of Harbin Normal University, China (Grant no. 11XYG-05 and no. 12XYS-04), and academic 

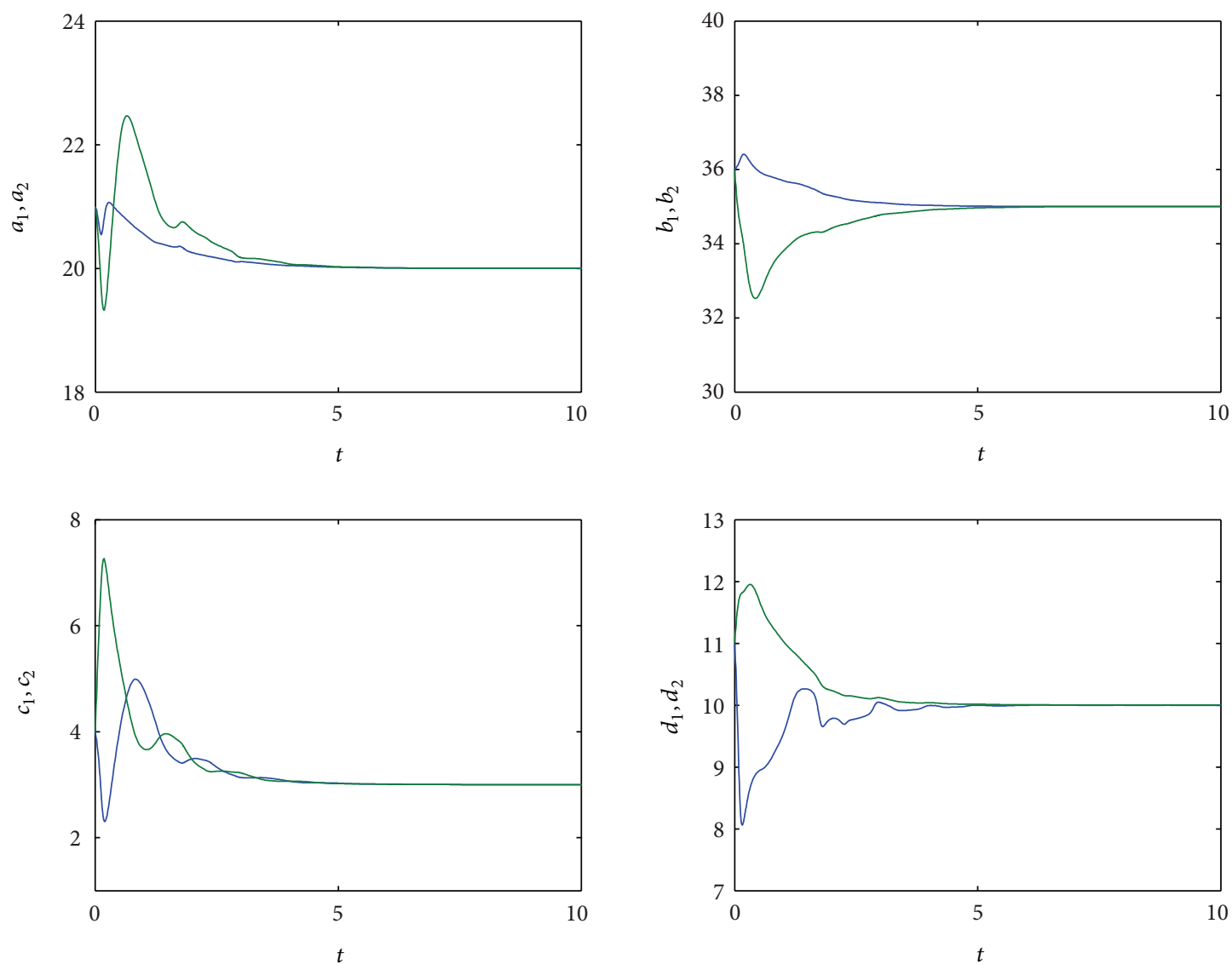

FIGURE 6: Curves of the estimated parameters of the drive-response systems (20) and (21) under the update law (26) and (27).
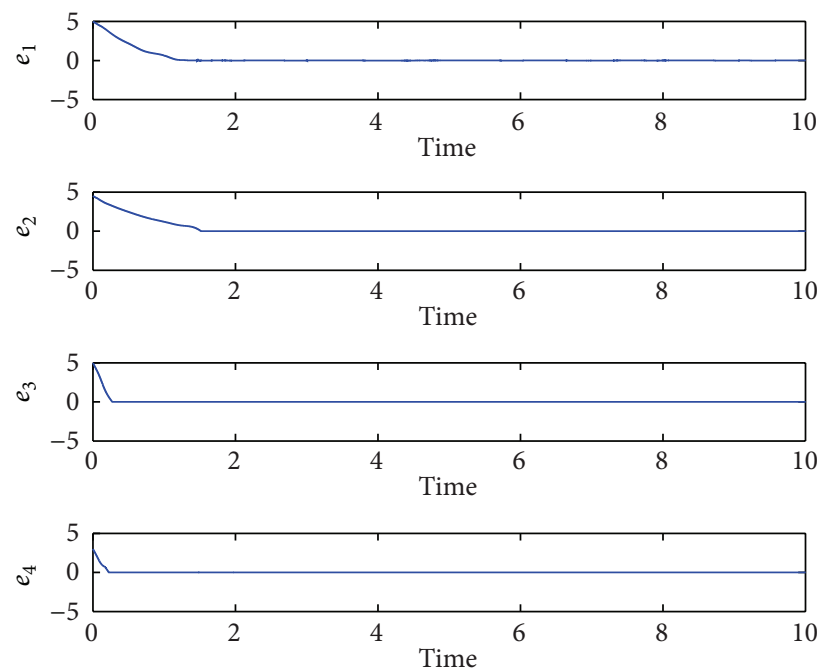

FIGURE 7: The MPS errors $e_{1}, e_{2}, e_{3}$, and $e_{4}$ of drive-response systems (20) and (21) with time-varying parameters. 
backbone program foundation for youth by Harbin Normal University (Grant no. KGB201222).

\section{References}

[1] L. M. Pecora and T. L. Carroll, "Synchronization in chaotic systems," Physical Review Letters, vol. 64, no. 8, pp. 821-824, 1990.

[2] S. Boccaletti, J. Kurths, G. Osipov, D. L. Valladares, and C. S. Zhou, "The synchronization of chaotic systems," Physics Reports, vol. 366, no. 1-2, pp. 1-101, 2002.

[3] A. S. Pikovsky, M. G. Rosenblum, G. V. Osipov, and J. Kurths, "Phase synchronization of chaotic oscillators by external driving," Physica D, vol. 104, no. 3-4, pp. 219-238, 1997.

[4] M. G. Rosenblum, A. S. Pikovsky, and J. Kurths, "From phase to lag synchronization in coupled chaotic oscillators," Physical Review Letters, vol. 78, no. 22, pp. 4193-4196, 1997.

[5] N. F. Rulkov, M. M. Sushchik, L. S. Tsimring, and H. D. I. Abarbanel, "Generalized synchronization of chaos in directionally coupled chaotic systems," Physical Review E, vol. 51, no. 2, pp. 980-994, 1995.

[6] R. Mainieri and J. Rehacek, "Projective synchronization in three-dimensional chaotic systems," Physical Review Letters, vol. 82, no. 15, pp. 3042-3045, 1999.

[7] G.-H. Li, "Generalized projective synchronization of two chaotic systems by using active control," Chaos, Solitons and Fractals, vol. 30, no. 1, pp. 77-82, 2006.

[8] G.-H. Li, "Modified projective synchronization of chaotic system," Chaos, Solitons and Fractals, vol. 32, no. 5, pp. 1786-1790, 2007.

[9] O. E. Rössler, "An equation for hyperchaos," Physics Letters A, vol. 71, no. 2-3, pp. 155-157, 1979.

[10] X. Wang and M. Wang, "A hyperchaos generated from Lorenz system," Physica A, vol. 387, no. 14, pp. 3751-3758, 2008.

[11] T. Gao, G. Chen, Z. Chen, and S. Cang, "The generation and circuit implementation of a new hyper-chaos based upon Lorenz system," Physics Letters A, vol. 361, no. 1-2, pp. 78-86, 2007.

[12] Y. Li, W. K. S. Tang, and G. Chen, "Generating hyperchaos via state feedback control," International Journal of Bifurcation and Chaos, vol. 15, no. 10, pp. 3367-3375, 2005.

[13] A. Chen, J. Lu, J. Lü, and S. Yu, "Generating hyperchaotic Lü attractor via state feedback control," Physica A, vol. 364, pp. 103110, 2006.

[14] M. T. Yassen, "Synchronization hyperchaos of hyperchaotic systems," Chaos, Solitons and Fractals, vol. 37, no. 2, pp. 465475, 2008.

[15] C.-H. Chen, L.-J. Sheu, H.-K. Chen et al., "A new hyper-chaotic system and its synchronization," Nonlinear Analysis: Real World Applications, vol. 10, no. 4, pp. 2088-2096, 2009.

[16] J. Huang, "Chaos synchronization between two novel different hyperchaotic systems with unknown parameters," Nonlinear Analysis: Theory, Methods and Applications, vol. 69, no. 11, pp. 4174-4181, 2008.

[17] M. M. Al-Sawalha and M. S. M. Noorani, "Adaptive anti-synchronization of two identical and different hyperchaotic systems with uncertain parameters," Communications in Nonlinear Science and Numerical Simulation, vol. 15, no. 4, pp. 1036-1047, 2010.

[18] C. Zhu, "Adaptive synchronization of two novel different hyperchaotic systems with partly uncertain parameters," Applied Mathematics and Computation, vol. 215, no. 2, pp. 557-561, 2009.
[19] E. E. Mahmoud, "Dynamics and synchronization of new hyperchaotic complex Lorenz system," Mathematical and Computer Modelling, vol. 55, no. 7-8, pp. 1951-1962, 2012.

[20] G. Fu, "Robust adaptive modified function projective synchronization of different hyperchaotic systems subject to external disturbance," Communications in Nonlinear Science and Numerical Simulation, vol. 17, no. 6, pp. 2602-2608, 2012.

[21] Y. Chen, X. Wu, and Z. Gui, "Global synchronization criteria for a class of third-order non-autonomous chaotic systems via linear state error feedback control," Applied Mathematical Modelling, vol. 34, no. 12, pp. 4161-4170, 2010.

[22] H. Du, Q. Zeng, C. Wang, and M. Ling, "Function projective synchronization in coupled chaotic systems," Nonlinear Analysis: Real World Applications, vol. 11, no. 2, pp. 705-712, 2010.

[23] H.-H. Chen, G.-J. Sheu, Y.-L. Lin, and C.-S. Chen, "Chaos synchronization between two different chaotic systems via nonlinear feedback control," Nonlinear Analysis: Theory, Methods and Applications, vol. 70, no. 12, pp. 4393-4401, 2009.

[24] J. H. Park, "Further results on functional projective synchronization of Genesiotesi chaotic system," Modern Physics Letters $B$, vol. 23, no. 15, pp. 1889-1895, 2009.

[25] T. H. Lee and J. H. Park, "Adaptive functional projective lag synchronization of a hyperchaotic Rössler system," Chinese Physics Letters, vol. 26, no. 9, Article ID 090507, 4 pages, 2009.

[26] J. Zheng, "A simple universal adaptive feedback controller for chaos and hyperchaos control," Computers and Mathematics with Applications, vol. 61, no. 8, pp. 2000-2004, 2011.

[27] J. H. Park, "Adaptive controller design for modified projective synchronization of Genesio-Tesi chaotic system with uncertain parameters," Chaos, Solitons and Fractals, vol. 34, no. 4, pp.11541159, 2007.

[28] J. H. Park, "Adaptive control for modified projective synchronization of a four-dimensional chaotic system with uncertain parameters," Journal of Computational and Applied Mathematics, vol. 213, no. 1, pp. 288-293, 2008.

[29] N. Jia and T. Wang, "Chaos control and hybrid projective synchronization for a class of new chaotic systems," Computers and Mathematics with Applications, vol. 62, no. 12, pp. 4783-4795, 2011.

[30] X. Mu and L. Pei, "Synchronization of the near-identical chaotic systems with the unknown parameters," Applied Mathematical Modelling, vol. 34, no. 7, pp. 1788-1797, 2010.

[31] S. Zheng, "Adaptive modified function projective synchronization of unknown chaotic systems with different order," Applied Mathematics and Computation, vol. 218, no. 10, pp. 5891-5899, 2012.

[32] Z. Wei, "Delayed feedback on the 3-D chaotic system only with two stable node-foci," Computers and Mathematics with Applications, vol. 63, no. 3, pp. 728-738, 2012.

[33] T. Botmart, P. Niamsup, and X. Liu, "Synchronization of non-autonomous chaotic systems with time-varying delay via delayed feedback control," Communications in Nonlinear Science and Numerical Simulation, vol. 17, no. 4, pp. 1894-1907, 2012.

[34] Y. Yu and H.-X. Li, "Adaptive hybrid projective synchronization of uncertain chaotic systems based on backstepping design," Nonlinear Analysis: Real World Applications, vol. 12, no. 1, pp. 388-393, 2011.

[35] F. Chen, L. Chen, and W. Zhang, "Stabilization of parameters perturbation chaotic system via adaptive backstepping technique," Applied Mathematics and Computation, vol. 200, no. 1, pp. 101-109, 2008. 
[36] M. P. Aghababa and A. Heydari, "Chaos synchronization between two different chaotic systems with uncertainties, external disturbances, unknown parameters and input nonlinearities," Applied Mathematical Modelling, vol. 36, no. 4, pp. 16391652, 2012.

[37] S. H. Hosseinnia, R. Ghaderi, A. Ranjbar N., M. Mahmoudian, and S. Momani, "Sliding mode synchronization of an uncertain fractional order chaotic system," Computers and Mathematics with Applications, vol. 59, no. 5, pp. 1637-1643, 2010.

[38] M. Hu, Y. Yang, and Z. Xu, "Impulsive control of projective synchronization in chaotic systems," Physics Letters A, vol. 372, no. 18, pp. 3228-3233, 2008.

[39] H. Hamiche, K. Kemih, M. Ghanes, G. Zhang, and S. Djennoune, "Passive and impulsive synchronization of a new fourdimensional chaotic system," Nonlinear Analysis: Theory, Methods and Applications, vol. 74, no. 4, pp. 1146-1154, 2011. 


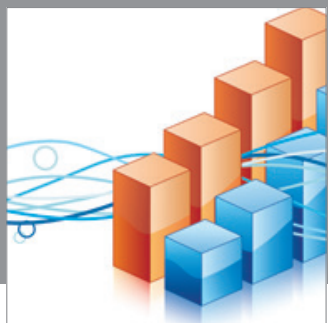

Advances in

Operations Research

mansans

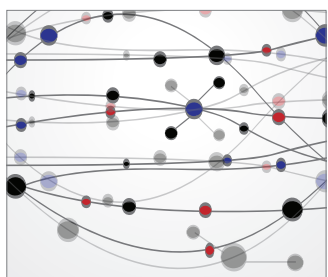

The Scientific World Journal
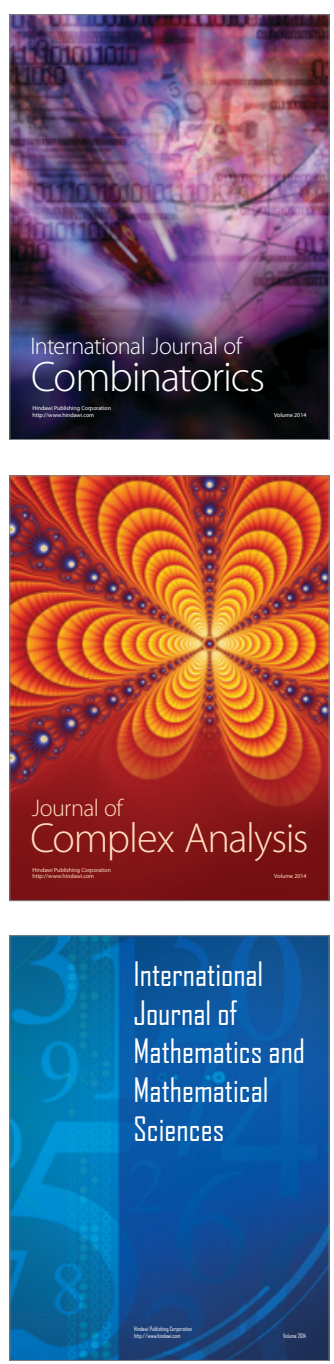
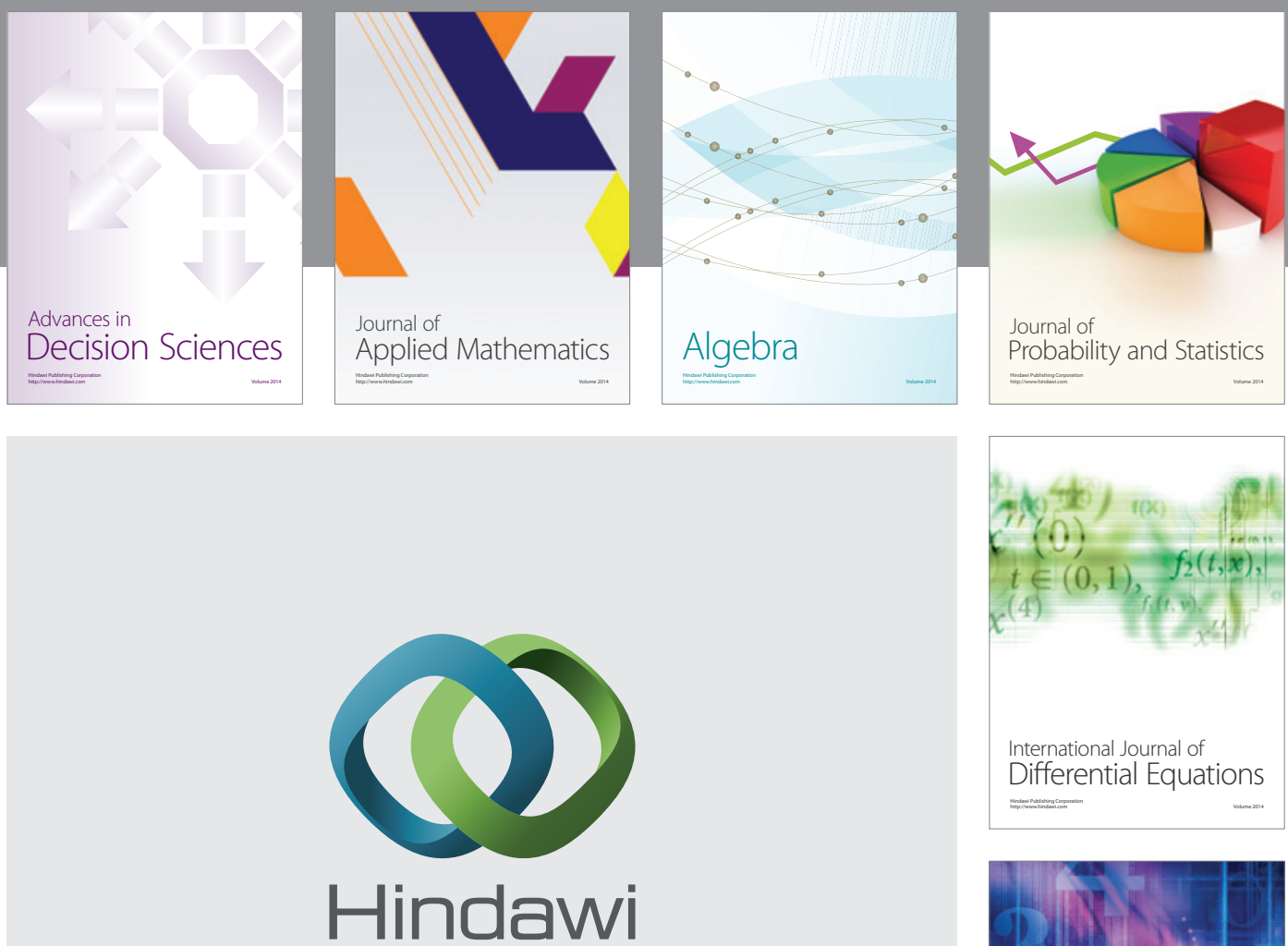

Submit your manuscripts at http://www.hindawi.com
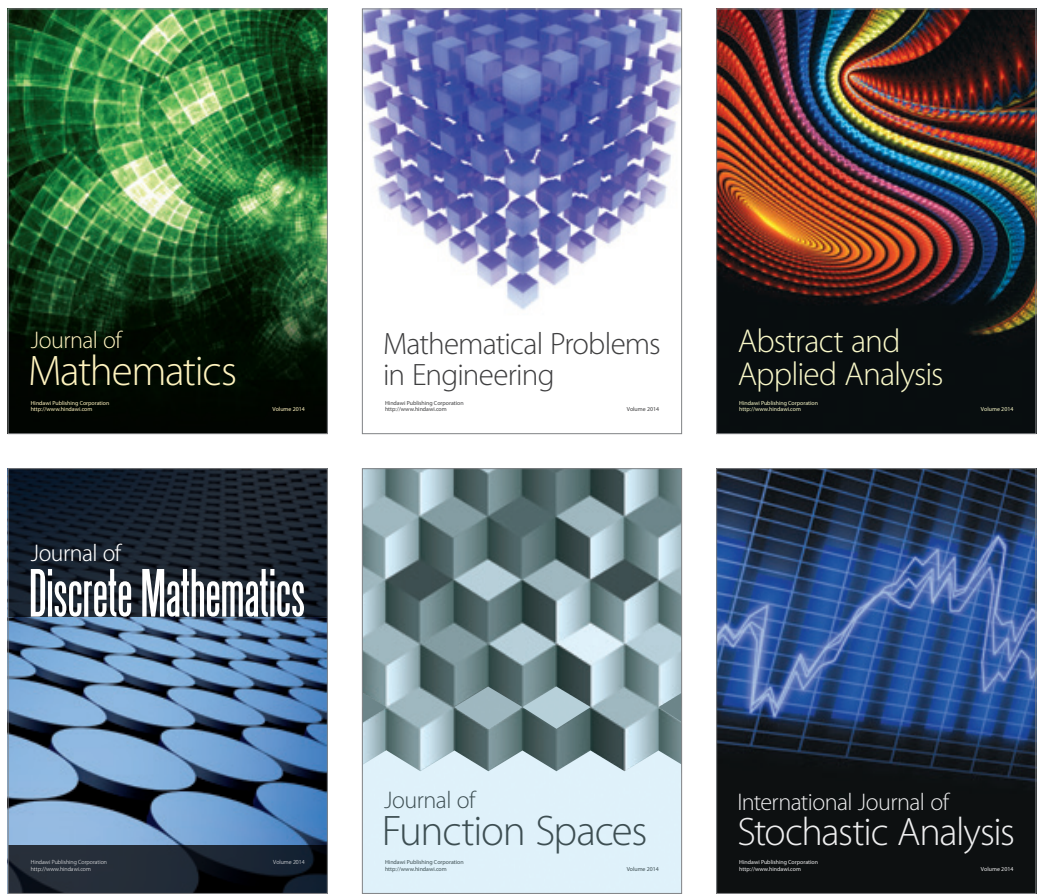

Journal of

Function Spaces

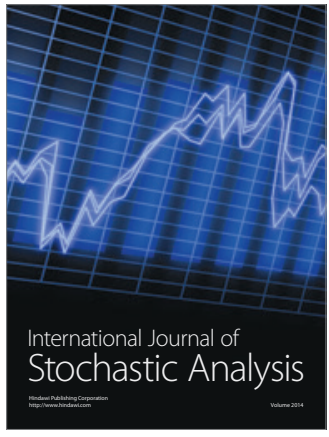

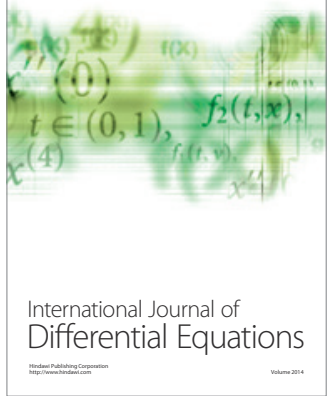
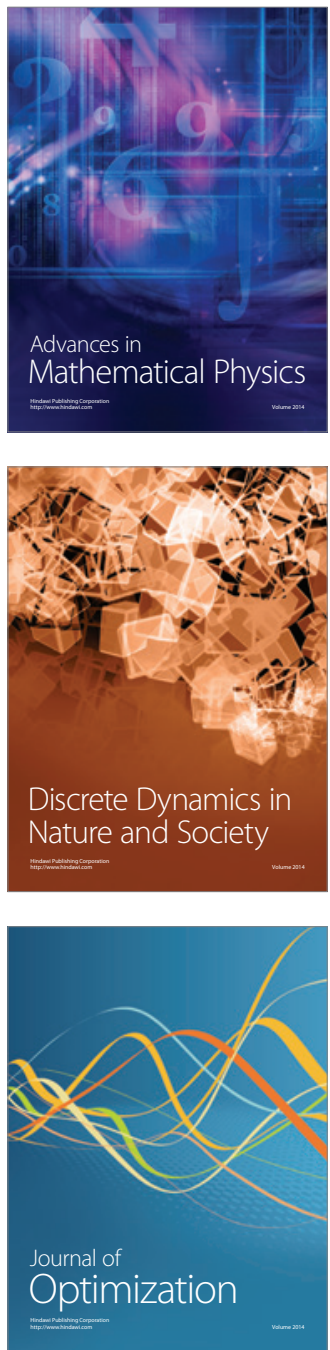